\title{
Asset Pricing under Information-processing Constraints
}

\author{
Yulei Luo* \\ Eric R. Young ${ }^{\dagger}$ \\ University of Hong Kong \\ University of Virginia
}

November 2007

\begin{abstract}
This paper studies the implications of limited information-processing capacity (also called "rational inattention") for asset pricing in a linear-quadratic permanent income model. It is shown that rational inattention lowers asset prices and raises expected excess returns by altering the dynamic responses of consumption to endowment shocks and increasing the volatility of consumption relative to the endowment.
\end{abstract}

JEL Classification Numbers: C61, D81, E21.

Keywords: Rational Inattention, Asset Pricing, Permanent Income.

\footnotetext{
* Corresponding author. School of Economics and Finance, University of Hong Kong, Hong Kong, email: yluo@econ.hku.hk.

${ }^{\dagger}$ Department of Economics, University of Virginia, Charlottesville, VA 22904, email: ey2d@virginia.edu.
} 


\section{Introduction}

The rational expectations hypothesis assumes that agents are endowed with infinite informationprocessing capacity, thereby allowing them to respond immediately and completely to changes in the economy. However, ordinary individuals in reality do not seem to have unlimited mental capacity, as mounting evidence suggests that they do not respond swiftly or thoroughly to all available information. Sims (2003) proposes rational inattention (RI) to capture this fact by assuming that agents only have finite processing capacity about the information on the state of the economy. He shows that RI can introduce realistic features such as sluggishness, randomness, and delays into the responses of economic variables to shocks.

This paper considers a simple rational inattention version of the permanent-income model as studied in Hall (1978) and examines the implications of RI for the pricing of multi-period securities. The focus of the PIH model is on the relationship between aggregate consumption and aggregate income in an environment in which output can be stored in the form of capital to smooth consumption over time. In contrast, the focus of the intertemporal asset pricing model is on the relationship between aggregate consumption and equilibrium asset prices. Following Hansen (1987) and Cochrane (chapter 2, 2005), we set up a model that is a combination of Hall's permanent income model and Lucas's asset pricing model. The model here can also be viewed as a parametric version of the intertemporal asset-pricing model as studied by Lucas (1978). We use the approach from Sims (2003) and Luo (2007) to solve the model with RI explicitly and then explore how RI affects asset prices within this framework.

Following Cochrane (2005), we decompose the price of a risky asset into a risk-neutral perpetuity and a risk adjustment component. RI alters asset pricing by increasing the size of the risk adjustment relative to the risk-neutral component, leading to a decline in the price of the asset (a rise in the risk premium required to hold that asset). This decline is driven by two key effects RI increases the volatility of the (perceived) movements in consumption and introduces persistence into consumption growth. The size of the price decline is negatively related to the channel capacity of the agents; agents with low channel capacity will require large premia to hold risky assets relative to those with unlimited capacity. 


\section{The Model}

The model is a simplified version of Hansen (1987)'s model, in which Hall's permanent income model and Lucas's asset pricing model are combined to examine the asset pricing implications of exogenous endowment shocks. In this section, we first derive the expression of (optimal) aggregate consumption in terms of the state variables by solving an otherwise standard PIH model with RI; we then price assets by treating the process of aggregate consumption that solves the RIPIH model as though it were an endowment process. Because we adopt the representative agent setup, equilibrium prices are shadow prices that leave the representative agent content with that endowment process.

\subsection{The Standard Permanent-Income Model}

A standard rational expectations (RE) version of the PIH model can be formulated as follows

$$
\max _{\left\{c_{t}\right\}} E_{0}\left[\sum_{t=0}^{\infty} \beta^{t} u\left(c_{t}\right)\right]
$$

subject to

$$
k_{t+1}=R k_{t}+e_{t}-c_{t},
$$

where $u\left(c_{t}\right)=-\frac{1}{2}\left(c_{t}-\bar{c}\right)^{2}$ is the utility function, $\bar{c}$ is the bliss point, Equation (2.2) represents a linear production technology, $c_{t}$ is consumption, $k_{t}$ is capital, $e_{t}$ is exogenous endowment with Gaussian white noise innovations, $\beta$ is the discount factor, and $R$ is the rate of return on capital. Let $\beta R=1$; then this specification implies that optimal consumption is determined by permanent income:

$$
c_{t}=(R-1) s_{t}
$$

where

$$
s_{t}=k_{t}+\frac{1}{R} \sum_{j=0}^{\infty}\left(\frac{1}{R}\right)^{j} E_{t}\left[e_{t+j}\right]
$$

is the expected present value of lifetime resources, consisting of physical capital plus human wealth. As noted in Cochrane (chapter 2, 2005), it is not a partial equilibrium result - it is a general equilibrium model with a linear production technology and an endowment process. As shown in Luo (2007), the above PIH model can be reduced to the univariate model with iid innovations to permanent income $s_{t}$ that can be solved in closed-form after introducing RI. Specifically, if $s_{t}$ is 
defined as a new state variable, we can rewrite the evolution equation of $s_{t}$ as

$$
s_{t+1}=R s_{t}-c_{t}+\zeta_{t+1}
$$

where the $t+1$ innovation $\zeta_{t+1}$ is

$$
\zeta_{t+1}=\frac{1}{R} \sum_{j=t+1}^{\infty}\left(\frac{1}{R}\right)^{j-(t+1)}\left(E_{t+1}-E_{t}\right)\left[e_{j}\right]
$$

This reduction is critically important for the RI model because multi-dimensional RI problems do not remain within the linear-quadratic-Gaussian class that can be solved analytically. ${ }^{1}$

Under the RE hypothesis, consumption growth can be written as

$$
\begin{aligned}
\Delta c_{t} & =\frac{R-1}{R}\left(E_{t}-E_{t-1}\right)\left[\sum_{j=0}^{\infty}\left(\left(\frac{1}{R}\right)^{j} e_{t+j}\right)\right] \\
& =(R-1) \zeta_{t},
\end{aligned}
$$

which relates the innovations to consumption to endowment shocks.

\subsection{Optimal Consumption under RI}

Following Sims (2003) and Luo (2007), the consumer's information-processing constraint can be characterized by the equation

$$
\mathcal{H}\left(s_{t+1} \mid \mathcal{I}_{t}\right)-\mathcal{H}\left(s_{t+1} \mid \mathcal{I}_{t+1}\right)=\kappa
$$

where $\mathcal{I}_{t}$ is the consumer's currently processed information, $\kappa$ is the consumer's channel capacity, $\mathcal{H}\left(s_{t+1} \mid \mathcal{I}_{t}\right)$ denotes the entropy of the state prior to observing the new signal at $t+1$, and $\mathcal{H}\left(s_{t+1} \mid \mathcal{I}_{t+1}\right)$ is the entropy after observing the new signal. The concept of entropy is from information theory, and it characterizes the uncertainty in a random variable. Hence, (2.7) means that the reduction in the uncertainty about the state variable gained from observing a new signal is bounded by $\kappa$. As shown in Sims $(2003), \mathcal{D}_{t}$ is a normal distribution $N\left(\widehat{s}_{t}, \sigma_{t}^{2}\right)$; as a result,

\footnotetext{
${ }^{1}$ One should interpret the operator $\left(E_{t+1}-E_{t}\right)$ as generating the difference between an expectation taken with information at time $t+1$ and that taken with information at time $t$. Luo and Young (2007) contains a discussion of the problems multi-state RI models present.
} 
(2.7) can be reduced to

$$
\log \left|\psi_{t}^{2}\right|-\log \left|\sigma_{t+1}^{2}\right|=2 \kappa
$$

where $\sigma_{t+1}^{2}=\operatorname{var}\left[s_{t+1} \mid \mathcal{I}_{t+1}\right]$ and $\psi_{t}^{2}=\operatorname{var}\left[s_{t+1} \mid \mathcal{I}_{t}\right]$ are the posterior variance and prior variance of the state variable, respectively. ${ }^{2}$

It is straightforward to show that in the univariate case $(2.8)$ has a steady state $\sigma^{2}=\frac{\omega_{\zeta}^{2}}{\exp (2 k)-R^{2}}$. In this steady state the consumer behaves as if observing a noisy measurement of permanent income $s_{t+1}^{*}=s_{t+1}+\xi_{t+1}$, where $\xi_{t+1}$ is the endogenous noise with variance $\lambda_{t}^{2}=\operatorname{var}\left[\xi_{t+1} \mid \mathcal{I}_{t}\right]$ determined by the usual updating formula of the variance of a Gaussian distribution based on a linear observation:

$$
\sigma_{t+1}^{2}=\psi_{t}^{2}-\psi_{t}^{2}\left(\psi_{t}^{2}+\lambda_{t}^{2}\right)^{-1} \psi_{t}^{2}
$$

Note that in the steady state $\sigma^{2}=\psi^{2}-\psi^{2}\left(\psi^{2}+\lambda^{2}\right)^{-1} \psi^{2}$, which can be solved to obtain $\lambda^{2}=$ $\left[\left(\sigma^{2}\right)^{-1}-\left(\psi^{2}\right)^{-1}\right]^{-1}$.

As shown in Luo (2007), the consumption function under RI is

$$
c_{t}=(R-1) \widehat{s}_{t},
$$

where the conditional mean $\widehat{s}_{t}$ evolves according to the Kalman filter equation

$$
\widehat{s}_{t+1}=(1-\theta) \widehat{s}_{t}+\theta\left(s_{t+1}+\xi_{t+1}\right)
$$

$\theta=\sigma^{2} / \lambda^{2}=1-1 / \exp (2 \kappa) \in[0,1]$ is the constant optimal weight on any new observation. Straightforward calculations imply that

$$
\Delta c_{t}=\theta(R-1)\left[\left(\frac{\zeta_{t}}{1-(1-\theta) R L}\right)+\left(\xi_{t}-\frac{\theta R \xi_{t-1}}{1-(1-\theta) R L}\right)\right]
$$

\section{Equilibrium Asset Prices under RI}

Note that in the model $R$ is the return on technology and is not yet the interest rate (the equilibrium rate of return on one-period claims to consumption). As proposed in Cochrane (chapter 2, 2005), we first find optimal consumption and then price one-period claims from the equilibrium consumption

\footnotetext{
${ }^{2}$ Note that here we use the fact that the entropy of a Gaussian random variable is equal to half of its logarithm variance plus some constant term.
} 
stream. Denoting the risk free rate by $R^{f}$, we have the following Euler equation:

$$
\frac{1}{R^{f}}=E_{t}\left[\beta \frac{u^{\prime}\left(c_{t+1}\right)}{u^{\prime}\left(c_{t}\right)}\right]=\beta E_{t}\left[\frac{\bar{c}-c_{t+1}}{\bar{c}-c_{t}}\right]=\beta=\frac{1}{R}
$$

where $E_{t}[\cdot]$ is the consumer's expectation operator conditional on his processed information at time $t$.

We can now use the basic pricing equation, $p=E[m x],{ }^{3}$ to compute the price of the stream of aggregate consumption (it is treated as the stream of endowments) as

$$
\begin{aligned}
p_{t} & =E_{t}\left[\sum_{j=1}^{\infty}\left(m_{t, t+j} c_{t+j}\right)\right] \\
& =\sum_{j=1}^{\infty}\left(\beta^{j} \frac{\bar{c} c_{t}-c_{t}^{2}-\operatorname{var}_{t}\left[c_{t+j}\right]}{\bar{c}-c_{t}}\right) \\
& =\frac{1}{R-1} c_{t}-\frac{1}{\bar{c}-c_{t}} \Xi
\end{aligned}
$$

where

$$
\Xi=\sum_{j=1}^{\infty}\left(\beta^{j} \operatorname{var}_{t}\left[c_{t+j}\right]\right)
$$

and we use the facts that

$$
\begin{aligned}
m_{t, t+j} & =\beta^{j} \frac{u^{\prime}\left(c_{t+j}\right)}{u^{\prime}\left(c_{t}\right)}=\beta^{j} \frac{\bar{c}-c_{t+j}}{\bar{c}-c_{t}}, \\
E_{t}\left[c_{t+j}^{2}\right] & =\operatorname{var}_{t}\left[c_{t+j}\right]+c_{t}^{2} .
\end{aligned}
$$

Denoting the risk-neutral component of the asset price by $p_{t}^{r n}$ and the risk-adjusted component by $p_{t}^{r c}$, we have

$$
p_{t}^{r n}=\frac{1}{R-1} c_{t}
$$

and

$$
p_{t}^{r c}=\frac{1}{\bar{c}-c_{t}} \sum_{j=1}^{\infty}\left(\beta^{j} \operatorname{var}_{t}\left[c_{t+j}\right]\right) .
$$

Expressions (3.1) yield the following implications. The first term in (3.1) is the risk-neutral component, the value of a perpetuity paying $c_{t}$. The second term is a risk-adjusted component,

\footnotetext{
${ }^{3}$ As argued in Cochrane (2005), this equation tells us only what the price should be given the joint distribution of consumption (the discount factor) and the asset payoff. We know $E[m x]$ after solving the PIH model given the state variables and can use them to determine the asset price $p$.
} 
which lowers the asset price relative to the risk-neutral level because $c_{t} \leq \bar{c}$. The following proposition states our key result.

Proposition 1. The risk-neutral component $p_{t}^{r n}$ of the asset price is independent of the degree of inattention, while the risk-adjusted component in the asset price $p_{t}^{r c}$ increases with the degree of inattention. The ratio of the risk-adjusted component under $R I$ to that under $R E$ is

$$
F \equiv \frac{p_{t}^{r c}(\theta<1)}{p_{t}^{r c}(\theta=1)}=\left(1+2 \frac{1-\theta}{\theta}\right) \frac{\theta}{1-R^{2}(1-\theta)} .
$$

Proof. Expression (2.12) can be rewritten as

$$
\Delta c_{t+1}=\rho_{c} \Delta c_{t}+\theta(R-1)\left(\varepsilon_{t+1}+\xi_{t+1}-R \xi_{t}\right),
$$

where $\rho_{c}=(1-\theta) R$. The variance of consumption growth is

$$
\sigma_{c}^{2}=\operatorname{var}\left[\Delta c_{t}\right]=\frac{\theta}{1-R^{2}(1-\theta)} \omega_{\zeta}^{2}
$$

Substituting (3.6) into $\sum_{j=1}^{\infty}\left(\beta^{j} \operatorname{var}_{t}\left[c_{t+j}\right]\right)$ yields

$$
\begin{aligned}
\Xi & =\sum_{j=1}^{\infty}\left(\beta^{j} \operatorname{var}_{t}\left[c_{t+j}\right]\right) \\
& =\sum_{j=1}^{\infty}\left(\beta^{j} \operatorname{var}_{t}\left[c_{t+j}-c_{t}\right]\right) \\
& =\beta \sigma_{c}^{2}+\beta^{2}\left(2 \sigma_{c}^{2}+2 \operatorname{cov}\left[\Delta c_{t+2}, \Delta c_{t+1}\right]\right)+\beta^{3}\left(\begin{array}{c}
3 \sigma_{c}^{2}+2 \operatorname{cov}\left[\Delta c_{t+2}, \Delta c_{t+1}\right] \\
+2 \operatorname{cov}\left[\Delta c_{t+3}, \Delta c_{t+1}\right]+2 \operatorname{cov}\left[\Delta c_{t+2}, \Delta c_{t+3}\right]
\end{array}\right)+\cdots \\
& =\left(\sum_{j=1}^{\infty}\left(j \beta^{j}\right)\right) \sigma_{c}^{2}++2 \beta^{2}\left[\rho_{c}+\beta\left(2 \rho_{c}+\rho_{c}^{2}\right)+\beta^{2}\left(3 \rho_{c}+2 \rho_{c}^{2}+\rho_{c}^{3}\right)+\cdots\right] \sigma_{c}^{2} \\
& =\left[\sum_{j=1}^{\infty}\left(j \beta^{j}\right)+2 \frac{\beta \rho_{c}}{1-\beta \rho_{c}} \frac{\beta}{(1-\beta)^{2}}\right] \sigma_{c}^{2} \\
& =\left(1+2 \frac{\beta \rho_{c}}{1-\beta \rho_{c}}\right) \frac{\beta}{(1-\beta)^{2}} \sigma_{c}^{2} \\
& =\left(1+2 \frac{1-\theta}{\theta}\right) \frac{\beta}{(1-\beta)^{2}}(R-1)^{2}\left(\frac{\theta}{1-R^{2}(1-\theta)} \omega_{\zeta}^{2}\right) \\
& =\left(\frac{2-\theta}{\theta}\right)\left(\frac{\theta}{1-R^{2}(1-\theta)}\right) R \omega_{\zeta}^{2}
\end{aligned}
$$


where we use the fact that $\beta R=1$. If $\theta=1, \Xi=R \omega_{\zeta}^{2}$. Hence,

$$
F=\frac{2-\theta}{\theta} \frac{\theta}{1-R^{2}(1-\theta)}
$$

Expression (3.8) clearly shows that RI reduces the asset price by two channels: First, RI increases the volatility of consumption growth $\sigma_{c}^{2}$; second, RI introduces persistence into consumption growth $\rho_{c} \cdot{ }^{4}$ Furthermore, given the expression of $\sigma_{c}^{2}$, it is obvious that the higher the volatility of the innovation to permanent income $\omega_{\zeta}^{2}$, the lower the price. Note that asset prices are also affected by both the persistence and volatility of the fundamental endowment shocks since $\omega_{\zeta}^{2}$ depends on them. We will discuss this in subsection 5 . It is immediate that we obtain the following corollary (under the mild parameter restriction $\left.\theta>\frac{R^{2}-1}{R^{2}}\right){ }^{5}$

Corollary 2. $F$ is decreasing and convex in $\theta$ and increasing and convex in $R$. The cross-partial is negative.

Proof. By direct calculation,

$$
\begin{gathered}
\frac{\partial F}{\partial \theta}=-\frac{R^{2}+1}{\left(1-R^{2}(1-\theta)\right)^{2}}<0 \\
\frac{\partial^{2} F}{\partial \theta^{2}}=\frac{2\left(R^{2}+1\right) R^{2}}{\left(1-R^{2}(1-\theta)\right)^{3}}>0 \\
\frac{\partial F}{\partial R}=\frac{2(\theta-2) R(\theta-1)}{\left(1-R^{2}(1-\theta)\right)^{2}}<0 \\
\frac{\partial^{2} F}{\partial R^{2}}=2(\theta-2)(\theta-1) \frac{1+3 R^{2}(1-\theta)}{\left(1-R^{2}(1-\theta)\right)^{3}}>0 \\
\frac{\partial^{2} F}{\partial R \partial \theta}=2 R \frac{2 \theta-3-R^{2}(1-\theta)}{\left(1-R^{2}(1-\theta)\right)^{3}}<0 .
\end{gathered}
$$

\footnotetext{
${ }^{4}$ By direct calculation

$$
\frac{\partial \sigma_{c}^{2}}{\partial \theta}=\frac{R^{2}-1}{\left(1-R^{2}(1-\theta)\right)^{2}}>0
$$

and

$$
\rho_{c}=0
$$

iff $\theta=1$.

${ }^{5}$ This restriction is needed in many linear-quadratic-Gaussian RI models to avoid anomalous results as channel capacity converges to 0; see Luo (2007).
} 
Figure 1 plots the relationship between the degree of attention and $F$; for example, when $\theta=0.67$ and $R=1.01$ we obtain $F=2$, meaning RI doubles the risk-adjusted component of asset prices (since $F=1$ if $\theta=1$ ). ${ }^{6}$ One implication of (3.9) is that the price of the asset is increasing with $\theta$ in the representative consumer context; that is, the equity premium is a decreasing function of channel capacity. Furthermore, the return on the linear technology also has effects on asset prices, as seen in Figure 1; the effect of $R$ is stronger when $\theta$ is small. The lower price in the RI economy reflects both the aggregate (macroeconomic) risk and the induced noise due to limited information-processing ability.

\section{Equilibrium Asset Returns under RI}

In this section we examine the implications of RI for asset returns. Given (3.1), (3.8), and (3.5), the expected asset return can be written as

$$
\begin{aligned}
E_{t}\left[R_{t, t+1}\right] & =E_{t}\left[\frac{p_{t+1}+c_{t+1}}{p_{t}}\right] \\
& =E_{t}\left[\frac{\frac{R}{R-1} c_{t+1}-\frac{1}{\bar{c}-c_{t+1}} \Xi}{\frac{1}{R-1} c_{t}-\frac{1}{\bar{c}-c_{t}} \Xi}\right] \\
& =\frac{E_{t}\left[\frac{R}{R-1} c_{t+1}-\frac{R}{\bar{c}-c_{t}} \Xi+\frac{R}{\bar{c}-c_{t}} \Xi-\frac{1}{\bar{c}-c_{t+1}} \Xi\right]}{\frac{1}{R-1} c_{t}-\frac{1}{\bar{c}-c_{t}} \Xi} \\
& =R+\frac{\frac{R}{\bar{c}-c_{t}}+E_{t}\left[\frac{1}{c_{t+1}-\bar{c}}\right]}{\frac{1}{R-1} c_{t}-\frac{1}{\bar{c}-c_{t}} \Xi} \Xi,
\end{aligned}
$$

where we use the fact that $c_{t}=E_{t}\left[c_{t+1}\right]$. To evaluate $E_{t}\left[\frac{1}{c_{t+1}-\bar{c}}\right]$ we approximate the concave function $\frac{1}{c_{t+1}-\bar{c}}$ around the conditional expectation of $c_{t+1}, E_{t}\left[c_{t+1}\right]$, to obtain $\frac{1}{c_{t+1}-\bar{c}} \simeq \frac{1}{E_{t}\left[c_{t+1}\right]-\bar{c}}-\left(E_{t}\left[c_{t+1}\right]-\bar{c}\right)^{-2}\left(c_{t+1}-E_{t}\left[c_{t+1}\right]\right)+\left(E_{t}\left[c_{t+1}\right]-\bar{c}\right)^{-3}\left(c_{t+1}-E_{t}\left[c_{t+1}\right]\right)^{2}$

\footnotetext{
${ }^{6}$ We choose this numerical parametrization because it is consistent with a 1 percent annual risk-free rate and Luo and Young (2007) found that $\theta=0.67$ implies a relative volatility of consumption to income roughly consistent with US data.
} 
taking the conditional expectation $E_{t}[\cdot]$ of both sides gives

$$
\begin{aligned}
E_{t}\left[\frac{1}{c_{t+1}-\bar{c}}\right] & =\frac{1}{E_{t}\left[c_{t+1}\right]-\bar{c}}+\left(E_{t}\left[c_{t+1}\right]-\bar{c}\right)^{-3} \operatorname{var}_{t}\left[\Delta c_{t+1}\right] \\
& =\frac{1}{c_{t}-\bar{c}}+\left(c_{t}-\bar{c}\right)^{-3} \operatorname{var}_{t}\left[\Delta c_{t+1}\right] \\
& \leq \frac{1}{c_{t}-\bar{c}}
\end{aligned}
$$

since $c_{t} \leq \bar{c}$. Using this result, we can establish an upper bound for the expected return as

$$
E_{t}\left[R_{t, t+1}\right] \leq R+\frac{(R-1)^{2} \Xi}{c_{t}\left(\bar{c}-c_{t}\right)-(R-1) \Xi}=\bar{R}_{t, t+1}
$$

Clearly, $\bar{R}_{t, t+1}$ is increasing with the degree of inattention because $\frac{\partial \Xi}{\partial \theta}=\frac{\partial F}{\partial \theta} R \omega_{\zeta}^{2}<0$.

The expected return on the asset is given by

$$
\begin{aligned}
E_{t}\left[R_{t, t+1}\right] & =R+\frac{\frac{R}{\bar{c}-c_{t}}-E_{t}\left[\frac{1}{\bar{c}-c_{t+1}}\right]}{\frac{1}{R-1} c_{t}-\frac{1}{\bar{c}-c_{t}} \Xi} \Xi \\
& =R+\frac{(R-1)^{2}-(R-1)\left(c_{t}-\bar{c}\right)^{-2} \sigma_{c}^{2}}{c_{t}\left(\bar{c}-c_{t}\right)-(R-1) \Xi} \Xi \\
& =R+\frac{R-1}{\left(\bar{c}-c_{t}\right)^{2}} \frac{\left(\bar{c}-c_{t}\right)^{2}-\frac{R-1}{R} \frac{\theta}{2-\theta} \Xi}{c_{t}\left(\bar{c}-c_{t}\right) /(R-1)-\Xi} \Xi
\end{aligned}
$$

where we use the fact that

$$
\sigma_{c}^{2}=\frac{\theta}{2-\theta} \frac{(1-\beta)^{2}}{\beta} \Xi
$$

Expression (4.5) shows that the expected excess return is increasing with the degree of inattention:

$$
\frac{\partial E_{t}\left[R_{t, t+1}-R\right]}{\partial \theta}<0
$$

because $\frac{\partial \Xi}{\partial \theta}<0$ and $\frac{\partial}{\partial \theta}\left(-\frac{\theta}{2-\theta}\right)<0$. Hence, RI can drive up the expected excess return of the asset by reducing the asset price. Note that (4.4) implies that RI raises the expected excess return, $E_{t}\left[R_{t, t+1}-R\right]$, holding $R$ and $\sigma_{c}^{2}$ constant.

\section{Applications to Some Particular Income Processes}

In this section we provide explicit formulae for two income processes - a stationary $\operatorname{AR}(1)$ and a difference-stationary $\mathrm{AR}(1)$ process. 


\subsection{AR(1) Endowment Process}

Suppose that the endowment follows an $\mathrm{AR}(1)$ process in levels:

$$
e_{t+1}-\bar{e}=\rho_{1}\left(e_{t}-\bar{e}\right)+\varepsilon_{t+1} .
$$

(2.6) implies that

$$
\zeta_{t}=\frac{1}{R-\rho} \varepsilon_{t}
$$

In this case,

$$
\Xi=F \frac{R}{\left(R-\rho_{1}\right)^{2}} \omega_{\varepsilon}^{2}
$$

which means that $\Xi$ increases with $\rho_{1}$ and $\omega_{\varepsilon}^{2}$. In other words, RI can amplify the impact of endowment shocks on the risk-adjusted component in asset prices and expected returns, and thus drive down asset prices and drive up expected returns.

\subsection{Difference-stationary Endowment Process}

Suppose that the endowment follows a difference-stationary process (an AR(1) in differences):

$$
e_{t+1}-e_{t}=\rho_{2}\left(e_{t}-e_{t-1}\right)+\varepsilon_{t+1} .
$$

(2.6) implies that

$$
\zeta_{t}=\frac{R}{\left(R-\rho_{2}\right)(R-1)} \varepsilon_{t}
$$

In this case,

$$
\Xi=F \frac{R^{3}}{\left(R-\rho_{2}\right)^{2}(R-1)^{2}} \omega_{\varepsilon}^{2},
$$

which means that $\Xi$ increases with $\rho_{2}$ and $\omega_{\varepsilon}^{2}$.

\section{Conclusion}

In this paper we examine the implications of limited information-processing capacity - rational inattention in the sense of Sims (2003) - for asset prices in an otherwise standard permanentincome model. We find that RI raises the risk-adjusted component of the asset price by increasing both the relative volatility and persistence of consumption growth. 


\section{References}

[1] Campbell, John (2003), "Consumption-Based Asset Pricing," in Constantinides, George, Milton Harris, and René Stultz, eds., Handbook of the Economics of Finance Volume 1B, Amsterdam: North-Holland, pp. 803-87.

[2] Cochrane, John (2005), Asset Pricing, Princeton University Press.

[3] Hansen, Lars (1987), "Calculating Asset Prices in Three Example Economies," in Truman F. Bewley ed., Advances in Econometrics, Fifth World Congress, Cambridge University Press.

[4] Hall, Robert E. (1978), "Stochastic Implications of the Life Cycle-Permanent Income Hypothesis: Theory and Evidence," Journal of Political Economy 86(6), pp. 971-87.

[5] Lucas, Robert Jr. (1978), "Asset Prices in an Exchange Economy," Econometrica 46(6), pp. 1429-445.

[6] Luo, Yulei (2007), "Consumption Dynamics Under Information Processing Constraints," forthcoming in Review of Economic Dynamics.

[7] Luo, Yulei and Eric R. Young (2007), "Rational Inattention and Aggregate Fluctuations," working paper.

[8] Mehra, Rajnish and Prescott, Edward (1985), "The Equity Premium: A Puzzle," Journal of Monetary Economics 15, 145-161.

[9] Sims, Christopher A. (2003), "Implications of Rational Inattention," Journal of Monetary Economics 50(3), pp. 665-90. 
The Effects of RI for Asset Prices

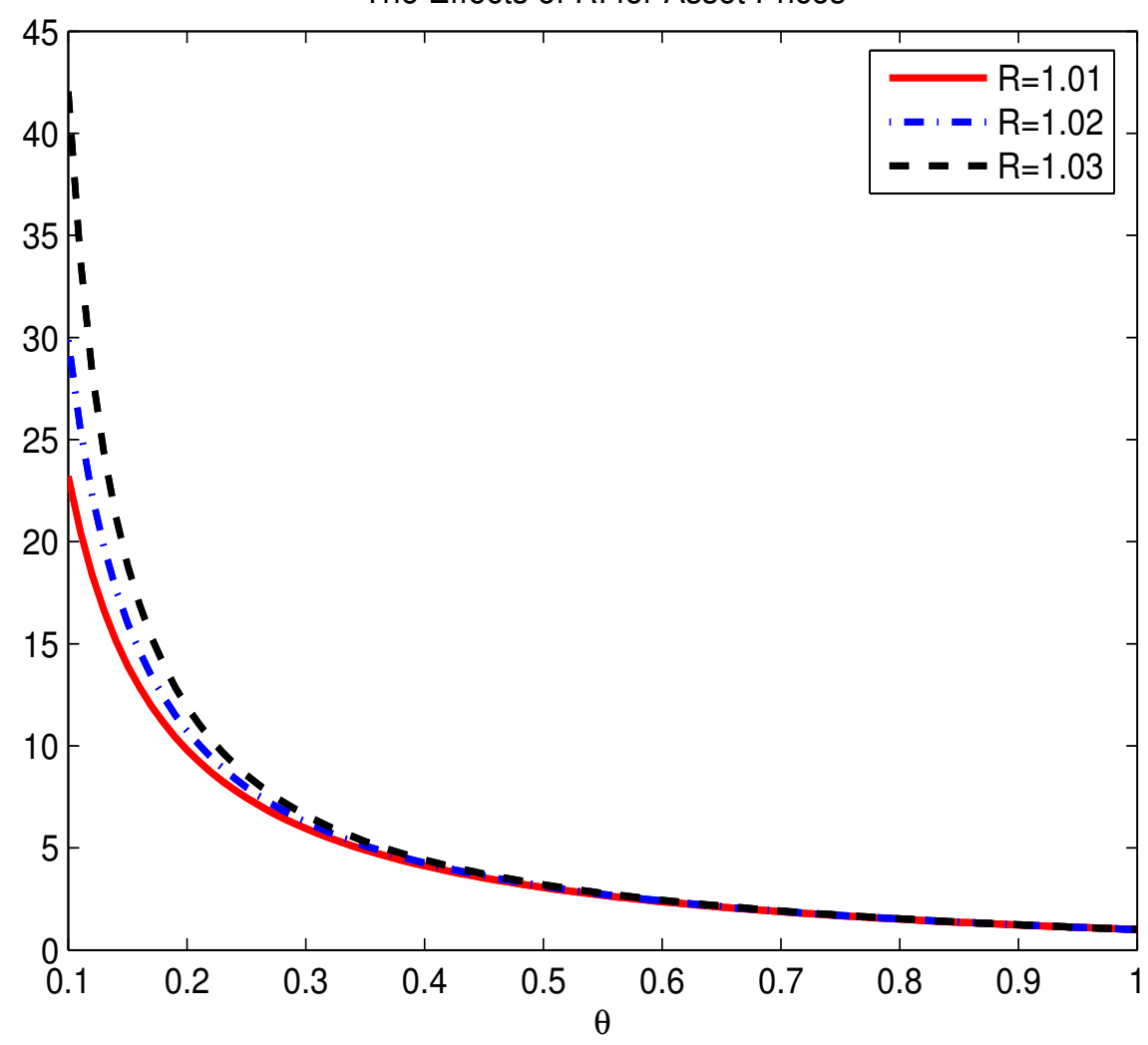

Figure 1 